\title{
The crystal orientation dependence of the elastic precursor shock strength in the PETN molecular explosive and the modeling of the steric hindrance to shear by molecular mechanics
}

\author{
J.J. Dick and J.P. Ritchie
}

Los Alamos National Laboratory, Group M-7, Mail Stop P952, Los Alamos, New Mexico 87545, U.S.A.

Résumé: Les niveaux des précurseurs élastiques des cristaux explosifs de pentrite (PETN) choques ont été mesurés pour des orientations [100], [101], [110] et [001], à l'aide d'instruments VISAR, sur des échantillons d'une épaisseur variant entre 3 et $6 \mathrm{~mm}$. Le niveaux de choc d'entrée était de $1.14 \mathrm{GPa}$. Les amplitudes du précurseur mesurées étaient respectivement de $0.38,0.58,0.98$ et $1.22 \mathrm{GPa}$ pour les quatre orientations. On a procédé à une modélisation par mécanique moléculaire, du cisaillement provoqué par le choc. Elle prédit correctement la dépendance de l'amplitude du précurseur par rapport à l'orientation des monocristaux pour les cas pris en compte, et explique l'observation du système de glissement $\{110\}<111>$ en déformation quasi statique, malgré la présence du vecteur de Burgers le plus long. Les résultats confirment l'importance de l'empêchement stérique du cisaillement dans le contrôle de la force suivant l'orientation dans certain cristaux moléculaires.

\begin{abstract}
The elastic precursor shock strengths of pentacisytnritol tetranitrate (PETN) explosive crystals were measured for [100], [101], [110], and [001] orientations using VISAR instrumentation for samples 3 to $6 \mathrm{~mm}$ thick. Input shock strength was $1.14 \mathrm{GPa}$. Measured precursor amplitudes were $0.38,0.58$, 0.98 , and $1.22 \mathrm{GPa}$, respectively, for the four orientations. Molecular mechanics modeling of slip in the ambient crystal was performed, analagous to the shock-induced shear for different orientations. The results show the observed dependence of the precursor amplitude on crystal orientation for the cases considered and explains why the $\{110\}<1 \overline{1} 1\rangle$ slip system is observed in quasistatic deformation in spite of having the longest Burgers vector. The results confirm the importance of steric hindrance to shear in controlling the orientation-dependent strength in some molecular crystals.
\end{abstract}

\section{INTRODUCTION}

In previous work it has been shown that the shock initiation sensitivity of pentaerythritol tetranitrate (PETN) depends strongly on crystal orientation. ${ }^{1}$ This effect was explained in terms of a geometric analysis of the steric hindrance to shear in the uniaxial strain of a shock wave. On further thought it seemed likely that this sterically hindered shear would also control the critical shear stress on the slip systems active during shock deformation. Critical shear stress on different slip systems can be inferred from measurements of elastic precursor shock strength as a function of crystal orientation. In descriptions of dislocations in terms of elasticity theory and simple, monatomic lattices, the preferred slip system is the one having the shortest Burgers vector on the plane having the widest interplanar separation. ${ }^{2}$ In these terms the preferred slip system for PETN in the tetragonal crystal system would be $\{110\}<001>$ (plane and direction). This is the dominant slip system observed in the tetragonal metals $\beta$-tin and $\beta$-uranium. ${ }^{3}$ However, $\{110\}\langle 1 \overline{1} 1\rangle$ is observed is quasistatic deformation of PETN. ${ }^{4}$ Based on our analyses and previous work we believe that this is because of the additional energetic barrier to shear in molecular 
crystals caused by steric hindrance. ${ }^{1,5,6}$ The analyses led us to predict that the crystals oriented so as to have strong steric hindrance to shear in the uniaxial strain of a plane shock wave would have high strength as displayed in the amplitude of the elastic precursor shock, and the crystals with weak hindrance would have weak strength.

We report on a series of plate impact experiments using VISAR instrumentation to test this steric hindrance hypothesis by measuring the elastic precursor shock strength as a function of crystal orientation. An input shock strength of $1.14 \mathrm{GPa}$ was used. Orientation dependence of elastic precursor strength has been studied in other materials(summarized in Ref. 7). This is the first complete study for a molecular crystal. There were some earlier studies in PETN, ${ }^{8,9}$ but they did not study the unhindered orientations.

In earlier work ${ }^{1}$ the steric hindrance to shear was analyzed in terms of the shear of rigid molecules past one another on opposite sides of the shear plane in the unit cell of the crystal. It showed a large contrast between hindered and unhindered orientations in terms of the number of close approaches between atoms of molecules on opposite sides of the shear plane. But it gave approaches as close as a few tenths of an angstrom which was clearly unphysical. In this paper results of a more realistic molecular mechanics calculations of the molecular interactions across the shear plane are presented. It appears to be the first application of molecular mechanics to the problem of shear in inelastic deformation. Estimates of the strength of the energy barrier to shear have been obtained for most of the slip systems of interest as well as some details about the molecular deformations involved.

\section{EXPERIMENTAL TECHNIQUE}

Measurements of particle velocity histories for PETN crystals of four orientations subjected to 1.14GPa shocks were obtained using a light-gas gun facility. Projectiles made of 2024 aluminum were impacted on aluminum-coated, +x-cut, quartz discs $50 \mathrm{~mm}$ in diameter and $5 \mathrm{~mm}$ thick. Quartz discs were used so that a sharp elastic shock would be transmitted to the PETN crystal. The PETN crystals were bonded to the quartz disc with a silicone elastomer. The crystals had typical lateral dimensions of 15 $\mathrm{mm}$. Details of crystal purity, perfection, and preparation were given in Ref. 10. Polymethylmethacrylate (PMMA, Mil. Spec. P-5425D, preshrunk) mirrored windows $12.7 \mathrm{~mm}$ in diameter and thickness were bonded to the PETN crystals with the elastomer. The crystal and PMMA window were bonded together with a silicone elastomer and surrounded with a casting of elastomer, epoxy, or epoxy loaded with 40 vol\% fused silica in order to reduce edge effects. The measurement system used was a dual, push-pull, VISAR system. ${ }^{11}$ The dual VISAR with different fringe constants prevents ambiguity in determining the particle-velocity jump at the shock when extra fringes must be added.

\section{EXPERIMENTAL RESULTS}

The main goal of the experiments was to measure elastic precursor strengths for two sterically unhindered and two hindered PETN crystal orientations. The two unhindered orientations were [100] and [101]. The notation [efg] means that the shock propagated in a direction normal to the $\{e f g\}$ plane. The hindered orientations were [110] and [001]. Experiments were attempted at two thicknesses for each orientation with an input shock strength of $1.14 \mathrm{GPa}$ on the plastic Hugoniot. This value was calculated using the MACRAME code ${ }^{12}$ using the known Hugoniots of 2024 aluminum, $x$-cut quartz, and PETN and an average projectile velocity of $0.296 \mathrm{~mm} / \mu \mathrm{s}$. The calculation also predicts a final particle velocity at the PETN/PMMA interface of $0.261 \mathrm{~mm} / \mu \mathrm{s}$. The wave profiles measured at the PETN/PMMA interface for the thick samples are displayed in Fig. 1.

Several features are noteworthy in the data as presented in Fig. 1. The elastic precursor strength increases with crystal orientation in the order [100], [101], [110] and [001]. For the [001] orientation the response is almost totally elastic with just a small amount of relaxation and a small plastic wave. In general the elastic precursor strength does not decrease with propagation distance indicating that any precursor attenuation must have occurred in less than $3 \mathrm{~mm}$ of run.

The average values of the elastic precursor stress and critical shear stress for [100], [101], [110], and [001] orientations are $0.38,0.58,0.98$, and $1.22 \mathrm{GPa}$, respectively, and the critical shear stresses are $0.11,0.16,0.21$, and $\geq 0.22 \mathrm{Gpa}$, respectively. There is a factor of 3 variation in precursor stress and a factor of 2 in the critical shear stress with orientation. Critical shear stresses were calculated by 

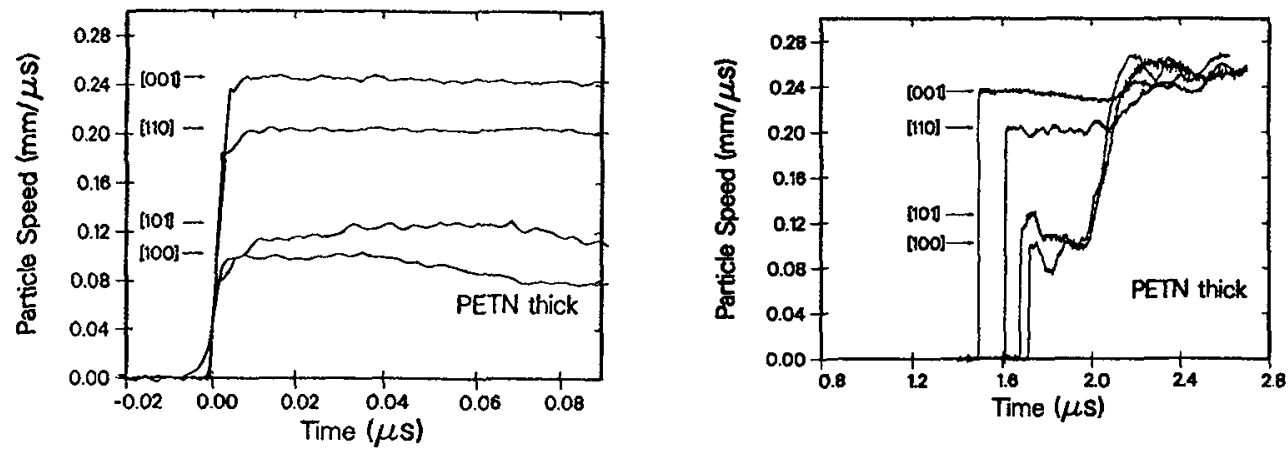

Figure 1. VISAR records at the PETN/PMMA interface. (a) Particle velocities vs time for the thick samples. Time origin is the arrival time of the elastic shock at the mirror in each case. Input shock strength was $1.14 \mathrm{GPa}(0.261 \mathrm{~mm} / \mu$ s at the interface). (b) Particle vs time for the thick samples showing the complete elastic and plastic wave profiles. Time origin is the time of shock entry into the sample.

assuming that the precursor shock is being supported by the slip system with the maximum resolved shear stress for that orientation. The ratios of shock stress to resolved shear stress were presented for these slip systems in PETN in Ref. 13. A complete presentation and discussion of the data will be given in another publication. ${ }^{14}$

The flow stress for PETN has been calculated from Vickers hardness measurements on crystal facets to be $0.050 \mathrm{GPa}^{15}$ The critical resolved shear stress on the $\{110\}<1 \overline{1} 1>$ preferred slip system calculated from the [100] elastic precursor shock strength is $0.11 \mathrm{GPa}$, a factor of two larger. The difference may be due to the much higher strain rates in shock experiments.

In Ref. 8 an elastic precursor strength of $1.97 \mathrm{GPa}$ was measured for an input shock strength of $2.64 \mathrm{GPa}$ in a sample $2.77 \mathrm{~mm}$ thick for a [110] shock orientation. In comparison, an elastic precursor strength of $1.01 \mathrm{GPa}$ was measured for an input shock strength of $1.14 \mathrm{GPa}$ for that orientation in this work for a sample $3.20 \mathrm{~mm}$ thick. Similar results were obtained for the [001] orientation. This indicates that the precursor shock strength and critical shear stress are strongly dependent on input shock strength, at least for the hindered orientations. For the [111] orientation an average elastic precursor stress of 1.06 GPa was measured for impact stresses from 1.8 to $3.0 \mathrm{Gpa}$ in samples about $3 \mathrm{~mm}$ thick by L. Soulard. ${ }^{9}$ No impact stress dependence was noted.

\section{STERIC HINDRANCE ANALYSIS}

Analysis of steric hindrance to plastic shear in the uniaxial strain of a plane shock wave was first investigated by the observation of crystal orientation dependence of the shock initiation sensitivity for initiation of detonation in PETN. ${ }^{1,13}$ It was observed that at the level of the crystal unit cell if molecules on one side of a slip plane could shear past molecules on the other side of the plane with few close approaches among atoms on different molecules, this correlated with insensitivity (no detonation or chemiluminescent emission). On the other hand, many close approaches between atoms on different molecules correlated with sensitivity.

\subsection{Geometric Analysis}

At first it was noted that orientations that could use the preferred $\{110\}$ slip plane were insensitive, whereas those that did not have that plane available were sensitive. ${ }^{13}$ The next step taken was to enumerate the number and type of close approaches (less than $1.7 \AA$ ) between atoms on molecules on opposite sides of the the shear plane during shear equivalent to that associated with the passage of an edge dislocation or simultaeous shear across the unit cell. The molecules were treated as rigid entities. The results along with closest approaches between atoms were given in Ref. 1 for the interactions with a moving body-centered molecule. Since there are two molecules per unit cell, the analysis was completed by including interactions with a corner molecule. These results are given in Ref. 14. Including the corner 


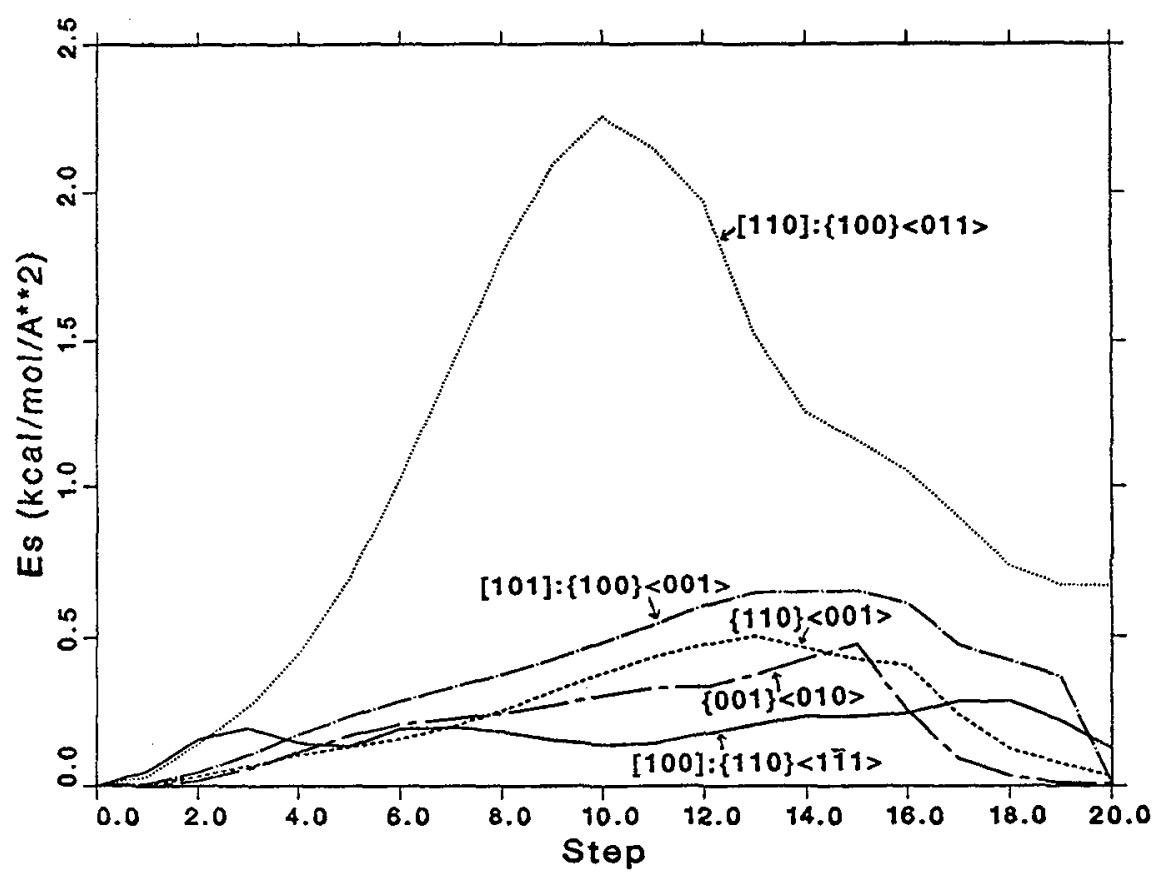

Figure 2. Steric energies calculated as described in the text at each step along the following slip systems: solid line, $\{110\}<1 \overline{1} 1>$ ([100] shocks, insensitive, quasistatic observed slip system); dash, $\{110\}<001>$ (slip system with shortest Burgers vector) ; chain-dash, $\{001]\}<010\rangle$ (largest value for closest approaches in geometric analysis); chain-dot, $\{100]\}<001\rangle$ ([101] shocks, insensitive); dot, $\{100\}<011>([110]$ shocks, sensitive).

molecule changes the order for [110] and [001] shocks in that now [001] has more interactions than [110], although [110] shocks cause the strongest initiation response. The order of increasing geometric hindrance is [100], [101], [110] and [001], the same as the order of increasing precursor strength.

The closest approaches are clearly unreasonable indicating the unphysical nature of the assumption of rigid molecules. Rotation about the $\mathrm{O}-\mathrm{NO}_{2}$ bond, for instance, requires very little energy. Nevertheless, the above geometric analysis appears to have some success in indicating in a qualitative manner the relative amount of steric hindrance to shear for the PETN orientations considered, at least in separating orientations into hindered and unhindered categories. The number and closeness of the interactions appears to give a rough indication of the energy barrier to shear due to steric hindrance.

\subsection{Molecular Mechanics Analysis}

4.2.1. The flexible nature of the PETN molecule The highly anisotropic nature and organic composition of PETN result in very different crystal properties from those of metals and inorganic ions that have been intensely studied in the material sciences. Consequently, concepts developed from those studies must be applied with care for PETN. PETN molecules interact with each other through much weaker non-bonding interactions and unlike many organic polymers, form high-quality molecular crystals.

One feature of PETN not previously addressed is the possibility of the individual molecules to deform easily. With the exception of the terminal oxygens of the nitro group, the atoms of the molecule are bonded together with single bonds. Internal rotation about an axis parallel with a single bond is usually quite easy. During edge dislocation motion the molecular environment changes and the additional energy required to initiate the motion is available. These factors can lead to substantial deformation of the PETN molecule that might significantly influence the ease of slip. Thus, more physical and quantitative analysis of steric hindrance can be achieved via molecular mechanics.

In order to gain insight into the factors described above, a series of molecular mechanics calculations have been performed. The results agree qualitatively with available experimental data and show that 
molecular deformations are an important consideration in determining the ease of slip in crystalline PETN.

\subsubsection{Molecular Mechanics Methods}

Calculations were performed using the program AMBER 4.0, ${ }^{16}$ which was developed and has been heavily applied in the study of biochemical systems. It has a molecular mechanics package in it that was used in this work. In this approach, the energy of a system of molecules is calculated from an equation having terms describing the energy cost associated with bond, angle, and dihedral angle distortions from the equilibrium values. These terms are calculated for the bonded atom pairs in a molecule. Intermolecular interactions are calculated using a Lennard-Jones interaction term. Standard values of equilibrium bond length and angles as well as the associated force constants have been determined previously ${ }^{16}$ for other systems. It was necessary to develop a number of additional parameters to describe the nitro group and the atomic charges in PETN. Details of the molecular mechanics equations, constants, and techniques used are given in Refs. 5 and 14.

A representative computational set-up consisted of two blocks, each made up of $3 \times 3 \times 2$ unit cells of PETN, that were constructed using the observed $x$-ray and neutron crystal coordinates. ${ }^{17}$ Each unit cell contains two molecules, so this system as a whole contained 72 molecules or 2088 atoms. Slip on other planes was examined using a similar setup. Using this initial setup, the geometry of the individual molecules were then energy optimized, subject to the constraint that the carbon atoms remain fixed. This constraint was employed to ensure that the model system resemble the real system and to minimize edge effects resulting from the small system size. The resulting geometry was then used in the slip calculations.

Slip was modeled by displacing one of the PETN blocks over the other along a single repeat vector in 20 incremental steps. Geometrical parameters of the molecules in the blocks, excepting those associated with carbons, were energy optimized at each step. The final geometry of one step was then rigidly displaced by the required increment to produce the starting geometry for the next. Again, the carbons were constrained to remain at their equilibrium lattice positions within each unit cell. Although such calculations are highly idealized, simple models frequently play an important role in illuminating basic concepts.

\subsubsection{Molecular Mechanics Results And Discussion}

Five slip systems were examined. Three of these were proposed to be stimulated by an initiating shock wave in orientations that were relatively sensitive (S) or insensitive (I) as determined in Ref. $1:\{110\}<1 \overline{1} 1>$, (I); $\{100\}<001>$, (I); and $\{100\}<011>$,(S). Another system examined was $\{110\}<001>$, which had been proposed as the primary slip system based upon the magnitude of the Burgers vector. ${ }^{13,18}$ In elementary dislocation theory the energy of a dislocation is proportional to the square of the magnitude of the Burgers vector (Ref. 2, pp. 80, 271). The $<001>$ Burgers vector is less than half as long as the $\langle 1 \overline{1} 1\rangle$. Finally, $\{001\}<010\rangle$ was examined because, among the slip systems on which a rigid molecule analysis was performed, this system resulted in the largest value for the minimum intermolecular atom-atom distance during the shear. ${ }^{5,14}$ It might have been proposed as the primary slip system on this basis.

Finally the maximum energy required for slip of the model lattices must be considered. In order to compare results with somewhat different lattices and numbers of molecules, a procedure was devised to calculate an energy per unit area taking into account different surface areas in each case and the overhanging ledges during the shear displacements (see Ref. 5,14). The calculations show large amounts of molecular deformation during the shear process. The energy barrier to shear has large contributions from electrostatic repulsion and overlap. Intramolecular bond rotation and angle strain also play an important role in determining the ease of slip. There is little bond-length distortion. The results obtained are shown in Figure 2. It is immediately evident that for the cases considered slip on $\{100\}<011\rangle$ is most difficult. This is in agreement with previous analysis and experiments. ${ }^{1}$ Remarkably, slip is found to be easiest on $\{110\}<1 \overline{1} 1\rangle$ in agreement with the previous geometric analysis ${ }^{1}$ and experimental observation. ${ }^{4}$ The energy difference between this system and the other three remaining ones is significant. 
However it is conceivable that reasonable variations in the potential function parameters could narrow the difference or even change its sign. Nonetheless, the energy differences calculated agree with experiment and the corresponding molecular deformations for slip on $\{110\}<1 \overline{1} 1\rangle$ are less than those found on the other slip systems. In addition, the order of increasing hindrance is the same as the order of the measured precursor strengths. These factors show that the ease of slip in crystalline PETN is markedly influenced by molecular deformations.

\section{REFERENCES}

[1] Dick J.J., Mulford R.N., Spencer W.J., Pettit D.R., Garcia E., and Shaw D.C., J. Appl. Phys. 70 (1991) 3572.

[2] Hirth J.P. and Lothe J., Theory of dislocations, 2nd ed. (Krieger, Malabar, FL, 1992) p. 271.

[3] Barrett C.S. and Massalski T.B., Structure of metals (McGraw-Hill, New York, 1966) p. 404.

[4] Gallagher H.G., Halfpenny P.J., Miller J.C., and Sherwood J.N., Phil. Trans. R. Soc. Lond. A339 (1992) 293.

[5] Ritchie J.P. in Tenth international symposium on detonation (1993), in press.

[6] Watanabe T. and Izumi K., J. Crystal Growth 46 (1979) 747.

[7] Johnson J.N., Jones O.E., and Michaels T.E., J. Appl. Phys. 41 (1970) 2330.

[8] Halleck P.M. and Wackerle Jerry, J. Appl. Phys. 47 (1976) 976.

[9] Soulard L., Thesis, "Etude du Monocristal de Pentrite Soumis a un Choc Plan," L'Universite de Haute-Alsace, 1990; Soulard L. and Bauer F., in Shock compression of condensed matter-1989, edited by Schmidt S.C., Johnson J.N., and Davison L.W. (Elsevier, Amsterdam, 1990) p. 817.

[10] Dick J.J., J. Appl. Phys. 53 (1982) 6161.

[11] Hemsing Willard F., Rev. Sci. Instrum. 50 (1979) 73.

[12] The MACRAME code is a shock characteristics code developed by a colleague, J. N. Fritz.

[13] Dick J.J., J. Appl. Phys. 44 (1984) 859.

[14] Dick J.J. and Ritchie J.P., submitted to J. Appl. Phys.

[15] Krishna Mohan V., Jyothi Bhasu V.C., and Field J.E., Ninth symposium (international) on detonation (Office of Naval Research, Arlington, VA, 1989) p. 1276; Hagan J.T. and Chaudri M.M., J. Mater. Sci. 10 (1977) 1055.

[16] Pearlman D.A., Case D.A., Caldwell J.C., Seibel G.L., Singh U.C., Weiner P., and Kollman P.A., AMBER 4.0, University of California, San Francisco, 1991.

[17] a) Conant J.W., Cady H.H., Ryan R.R., Yarnell J.L., and Newsam J.M. , LA-7756-MS, December 1979. b) Trotter J., Acta. Cryst. 15 (1963) 698.

[18] Halfpenny P.J., Roberts K.J., and Sherwood J.N., J. Mater. Sci. 19 (1984) 1629. 